\title{
FREE CURING LIGHT
}

GC UK is offering a free Silverlight cordless, LED curing lamp with Fuji capsules. Choose from Fuji II LC, Fuji IX or a combination of the two. Your usual dealer will be able to provide you with full details.

Silverlight is a cordless, flexible LED curing lamp that can be taken from chair to chair. This extremely lightweight LED curing unit is reliable for curing a $2 \mathrm{~mm}$ thick layer in only ten seconds, which offers a choice between two operating modes: 'Fast' and 'Slow'. Thanks to its high performance level and the long-lasting lithium-ion battery, it will run for 320' cycles of ten seconds each in succession; recharging takes only 120 minutes and its output can be checked on the built-in radiometer.

- The Silverlight's sophisticated optical element focuses the light into the coherent fibre optics

- 'Fast' mode: ten seconds

- 'Slow' mode: 20 seconds with a progressive increase of the light intensity reaching its peak after three seconds

- Light Intensity: Minimum $1200 \mathrm{~mW} / \mathrm{cm}^{2}$ - Maximum $1500 \mathrm{~mW} / \mathrm{cm}^{2}$

- Spectra wavelength comprised between $440 \mathrm{~nm}$ and $480 \mathrm{~nm}$ with a peak at $460 \mathrm{~nm}$ (effective on camphorquinone)

- Lithium-ion battery with no memory effect and a charging time of only 120 minutes with a capacity sufficient for 320 ten-second cycles (extra battery is not included) - Smooth and compact surface makes the unit easy to disinfect; the rotating $\left(360^{\circ}\right)$ fibreglass light guide and optical protection are sterilisable at $135{ }^{\circ} \mathrm{C}$.

For further information

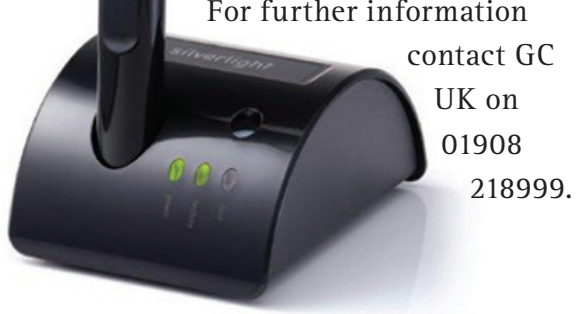

\section{A TOP SPLINTING MATERIAL}

Dentapreg are fibre-reinforced composite strips pre-impregnated with biocompatible light-curing resin. They are indicated for splinting of teeth in situations where no tooth preparation is necessary, including periodontal and post-traumatic stabilisation and orthodontic retention. They are also recommended for anterior and

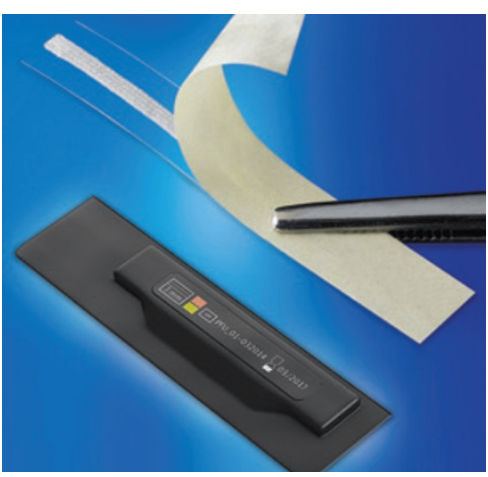

posterior bridges, denture repairs and strengthening of large restorations.

Dentapreg is fast and easy to use, minimally invasive and represents a reliable short to long-term solution. In contrast to other products using dry fibres, Dentapreg strips are pre-impregnated, pliable and do not require pre-soaking in the practice. A slight tackiness of the resin helps hold the strip in place during adaptation and no special tools are required. All this makes the procedures simple and time and cost efficient. Step-by-step educational videos are available on the UKdentistry.co.uk website, as well as a demonstration video showing how to create single visit tooth coloured fibre reinforced adhesive bridges.

Dentapreg was chosen the 'Top Splinting Material' and awarded the 'Editors' Choice' status by the US-based Dental Advisor magazine. The strips are available in different lengths and options and come in boxes of 1, 3 or 8 strips from only £30 incl. For more information or to order, visit www.UKdentistry.co.uk or call 02078710727.

\section{NEW PENSION PROTECTION AVAILABLE}

NHS dentists with pension benefits valued at more than $£ 1.25$ million are likely to benefit from HMRC's Individual Protection as of 18 August 2014. Dentists with NHS pension 'added years', personal pensions and those with deferred benefits can also take advantage of the newly available protection.
By having Individual Protection you are able to set your Lifetime Pension Allowance up to a maximum of $£ 1.5$ million, potentially saving a tax charge of $£ 137,500$.

For more information contact pfmdental on 08452414480 or visit www.pfmdental.co.uk.

\section{A DIAGNOSTIC TOOL WITH IMPRESSION MAKING CAPABILITIES}

Professor François Duret, the inventor of CAD/CAM in dentistry, has combined video image capture and satellite mapping technologies to develop the Condor scan system much more than an impression tool.

This scanning device has moved on from simple impression making, offering diagnostic, patient education and peer-to-peer communication tools. The Condor is best viewed as a diagnostic tool with state-of-the-art impression making capabilities.

Self-calibrating, Condor is ready to use when required and is small enough to be housed in any standard delivery unit. Indeed, the Condor handpiece is the same size and weight as a slow-speed handpiece and can be held in the familiar 'pen grip'. Furthermore, the Condor handheld scanner is not hardware-dependent, so if the handpiece is dropped or damaged then you can simply plug in a replacement handpiece and carry on.

With Condor, two cameras produce a 3D 'true colour' video of the scanned surface using satelliteimaging technology therefore the image produced is virtually identical to the clinical presentation. The Condor scan is in the process of being equipped with a malignancy detection function using intraoral fluorescent dyes, plus it will have a caries detection function using fluorescent dyes.

The Condor scan system was launched by First Stop Dental at October's BDIA Dental Showcase. To find out more, visit www.firststopdental.com.

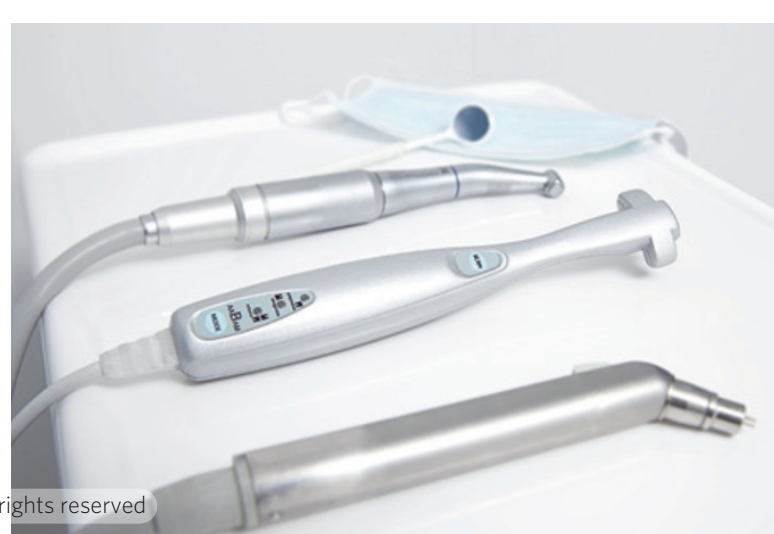

